\title{
Validación de la versión autoadministrada del PRIME-MD para diagnósticos psiquiátricos en atención primaria.
}

Validation and utility of a self-report version of PRIME-MD.

Spitzer RL, Kroenke K, Williams JBW and the Patient Health Questionnaire Primary Care Study Group. JAMA 1999; 282: 1737-44

\section{Objetivo}

Determinar si la versión autoadministrada del cuestionario de salud del paciente del PRIME-MD tiene validez y utilidad para el diagnóstico de trastornos psiquiátricos en atención primaria.

\section{Diseño}

Estudio de corte transversal.

Lugar

Se realizó en 8 clínicas de atención primaria de Estados Unidos.

\section{Pacientes}

Ingresaron al estudio 3000 pacientes adultos atendidos por 62 médicos de atención primaria (21 internistas generales y 41 médicos de familia).

\section{Descripción del test a evaluar}

El PRIME-MD (Primary Care Evaluation of Mental Disorders), instrumento para el diagnóstico de trastornos psiquiátricos en atención primaria, se componía originalmente de dos partes. El primer componente autoadministrado: cuestionario del paciente ( $\mathrm{PQ}$ ) con preguntas sobre 5 áreas de diagnóstico (depresión, ansiedad, trastornos de alimentación, alcoholismo y somatizaciones) y una pregunta relacionada con la salud general. El segundo componente: guía de evaluación clínica (CEG) que el médico de atención primaria administraba si en algún área diagnóstica del primer cuestionario el paciente respondía en forma positiva. El tiempo promedio de realización fue de 8.4 minutos. Este instrumento determinaba presencia o ausencia de 18 trastornos (distintos cuadros depresivos; distimia; trastorno bipolar; pánico y distintos trastornos de ansiedad; probable abuso/dependencia de alcohol; distintos trastornos de alimentación; somatización y otros trastornos somatomorfos e hipocondría).

Los dos componentes originales fueron combinados en un cuestionario único de tres páginas totalmente autoadministrado por el paciente: $\mathrm{Pa}$ tient Health Questionnaire (PHQ). El médico aplicó algorritmos diagnósticos, verificando las respuestas positivas. Los diagnósticos finales del PHQ fueron 8: depresión mayor, otros trastornos depresivos, pánico, otros trastornos de ansiedad, probable abuso/dependencia de alcohol, somatización, bulimia y otros trastornos de la alimentación.

\section{Descripción del test de referencia}

Se realizó una evaluación telefónica por profesionales de salud mental dentro de las 48 horas de completar el cuestionario y así se definía el diagnóstico psiquiátrico.

Además se midió estado funcional con SF-20, días de incapacidad, utilización de servicios médicos y derivaciones y/o tratamientos instaurados.

\section{Medición de resultados principales}

Sensibilidad, Especificidad. Acuerdo entre observadores medido con el índice kappa*.

\section{Resultados}

De los 3000 pacientes ingresados, 825 (28\%) presentaron un diagnóstico $\mathrm{PHQ}$ positivo. Se realizó evaluación psiquiátrica en 585 de los 3000 pacientes. De esos 585; 170 (29\%) presentó diagnóstico PHQ positivo. El grado de concordancia entre ambas evaluaciones (para cualquier diagnóstico psiquiátrico) presentó un kappa de 0,65. La exactitud global fue de $85 \%$ (IC95\%=82-88), con sensibilidad de 75\% (IC95\%=69-81) y especificidad del $90 \%$ (IC95\%=87-93). Los pacientes con diagnóstico positivo por PRIME-MD presentaron mayores valores de incapacidad funcional, días de incapacidad y utilización de servicios $(p<0,001)$. El tiempo requerido por el médico fue menor a 3 minutos en el $85 \%$ de los pacientes. El $80 \%$ de los médicos reportó que el uso rutinario del instrumento sería útil. Indicaron nuevos tratamientos en el $32 \%$ de los pacientes con diagnóstico positivo.

\section{Conclusiones}

El diagnóstico de trastornos psiquiátricos utilizando una versión autoadministrada del PRIME-MD presentó validez comparable a la versión original administrada en su totalidad por el médico de atención primaria.

\section{COMENTARIO}

Los pacientes que concurren a consultorios de atención primaria presentan altas prevalencias de desórdenes mentales. Se describieron dificultades para el diagnóstico de estos cuadros por médicos de atención primaria. ${ }^{1}$ Spitzar y col. ${ }^{2}$ realizaron la validación del PRIME-MD original (PC y CEG) que presentó un kappa de 0,71; una exactitud global del $88 \%$ (IC95\% 83-89\%), sensibilidad $=83 \%$ (IC95\% 78-88\%) y especificidad $=$ $88 \%$ (IC95\% 84-92). El uso en la práctica diaria del PRIME-MD original se vio limitado por el tiempo de administración de la guía de aplicación clínica. El presente trabajo intenta validar una adaptación de dicho instrumento pero totalmente autoadministrado por el paciente.

Al estudiar un instrumento se analizan fundamentalmente dos puntos: reproducibilidad y validez. En este trabajo no se analizó la reproducibilidad del instrumento. La validez se reportó con sensibilidad y especificidad. Se utilizó la entrevista telefónica como test de referencia o "gold standard". Los autores justificaron esta elección citando artículos que demostraron comparabilidad de la misma con la entrevista cara a cara. ${ }^{2}$ Una limitación del presente estudio es el no haber utilizado el gold stan- dard a todos los participantes (sólo lo hicieron en 585 de los 3 000). De todos modos se realizó una comparación de las características en los dos grupos y no parecían diferentes. La validez de construcción del cuestionario se analizó en base a la muestra en que se validó el PRIME-MD original, aunque sería más apropiado comparar los resultados de ambos instrumentos administrados a los mismos pacientes.

Para aplicar el instrumento en castellano se requiere validar la traducción, para asegurar su buen funcionamiento en otro idioma y/o cultura. Bonicatto y col. ${ }^{3}$ adaptaron al castellano el PRIME-MD original (PQ y CEG). Se administró a 122 pacientes en los que describen una adecuada aceptación del instrumento por parte de los profesionales y los pacientes. Aún no se cuenta con una traducción de la versión autoadministrada (PHQ). La información disponible resulta alentadora. Parece razonable pensar que en breve dispondremos de un instrumento práctico y en castellano para diagnosticar trastornos psiquiátricos en atención primaria. Esto podrá ayudar a prestarle más atención y mejor tratamiento a estos problemas tan importantes, prevalentes, y muchas veces no tratados.

\section{*Ver glosario}

Dra. Marina Khoury.

Servicio de Clínica Médica. Centro Medicus. Capital Federal. Docente de Metodología de la Investigación. Centro de Investigaciones Epidemiológicas. Academia Nacional de Medicina.

\section{Referencias}

1. Andersen SM Harthorn $\mathrm{BH}$. The recognition, diagnosis and treatment of mental disorders in a primary care practice. Med Care 1989-27:869-886.

2.Spitzer RL, Williams JBW, Kroenke K et al.: Utility of a neew procedure for diagnosing mental disorders in primary care: the PRIME-MD 1000 study. JAMA $1994 ; 272: 1749-56$.

3. Bonicatto S, Gargoloff P, Tutor C, Lucero S, Güenaga F, Torino D: Diagnóstico de trastornos mentales en atención primaria. Adaptación de un procedimiento de tamizaje. Revista Acta Psiquiá trica y Psicológica Latinoamericana. Sept 1999; 45(3):223-234. 